\title{
Efeitos fotobiomoduladores das luzes vermelha, azul e verde sobre o colágeno durante o processo de cicatrização de queimaduras cutâneas: uma revisão integrativa
}

\author{
Photobiomodulatory effect of red, blue and green lights on collagen during the healing process of
}

skin burns: an integrative review

Efecto fotobiomodulador de las luces rojas, azules y verdes sobre el colágeno durante el processo de curación de las quemaduras cutáneas: uma revisión integradora

Recebido: 10/02/2021 | Revisado: 11/02/2021 | Aceito: 25/02/2021 | Publicado: 03/03/2021

\author{
Alana Olivia Nascimento de Souza \\ ORCID: https://orcid.org/0000-0002-8015-1508 \\ Universidade Estadual da Paraíba, Brasil \\ E-mail: olivialanasouza@gmail.com \\ Maria Helena Chaves de Vasconcelos Catão \\ ORCID: https://orcid.org/0000-0001-7681-3225 \\ Universidade Estadual da Paraíba, Brasil \\ E-mail:mhelenact@zipmail.com.br
}

\begin{abstract}
Resumo
Introdução: A cicatrização de feridas é um mecanismo que envolve uma sequência de eventos moleculares com o objetivo de reparo tecidual. Neste sentido, destaca-se o uso fototerapia LED e laser devido as suas características bioestimulantes para a célula durante o processo de reparo. Objetivo: realizar uma revisão de literatura com o intuito de observar o efeito fotobiomodulador das luzes vermelha, azul e verde sobre o colágeno durante o processo de cicatrização de queimaduras. Metodologia: trata-se de uma revisão de literatura do tipo integrativa, realizada nas bases de dados Pubmed, Web of Science e Lilacs. Resultados: entre os 1721 estudos encontrados, somente 18 artigos, publicados entre os anos de 2011 a 2020, foram utilizados dos resultados. Conclusão: A fotobiomodulação das luzes vermelha, azul e verde no processo de reparo de queimaduras cutâneas mostrou resultados satisfatórios principalmente em relação a produção de colágeno, angiogênese, redução da inflamação, redução do tamanho da lesão e estímulo de fibroblastos.
\end{abstract}

Palavras-chave: Fototerapia; Terapia de luz de baixa intensidade; Queimaduras; Cicatrização.

\begin{abstract}
Introduction: Wound healing is a mechanism that involves a sequence of molecular events for the purpose of tissue repair. In this sense, the use of LED and laser phototherapy stands out due to its bio-stimulating characteristics for a cell during the repair process. Objective: to carry out a literature review in order to observe the photobiomodulator effect of red, blue and green lights on collagen during the healing process of burns. Methodology: this is an integrative literature review, carried out in the Pubmed, Web of Science and Lilacs databases. Results: among the 1,721 studies found, only 18 articles, published between 2011 and 2020, were used for the results. Conclusion: The photobiomodulation of red, blue and green lights in the skin burn repair process satisfactory results mainly in relation to collagen production, angiogenesis, reduction of inflammation, reduction of the size of the lesion and stimulation of fibroblasts.
\end{abstract}

Keywords: Phototherapy; Low intensity light therapy; Burns; Healing.

\section{Resumen}

Introducción: La cicatrización de heridas es un mecanismo que involucra una secuencia de eventos moleculares con el propósito de reparar los tejidos. En este sentido, el uso de fototerapia con LED y láser destaca por sus características bioestimulantes para una célula durante el proceso de reparación. Objetivo: realizar una revisión de la literatura para observar el efecto fotobiomodulador de las luces roja, azul y verde sobre el colágeno durante el proceso de curación de las quemaduras. Metodología: se trata de una revisión integradora de la literatura, realizada en las bases de datos Pubmed, Web of Science y Lilacs. Resultados: de los 1.721 estudios encontrados, solo se utilizaron para los resultados 18 artículos, publicados entre 2011 y 2020. Conclusión: La fotobiomodulación de luces rojas, azules y verdes en el proceso de reparación de quemaduras cutáneas resulta satisfactoria principalmente en relación a la producción de colágeno, angiogénesis, reducción de la inflamación, reducción del tamaño de la lesión y estimulación de los fibroblastos.

Palabras clave: Fototerapia; Terapia de luz de baja intensidade; Queimaduras; Curación. 


\section{Introdução}

A cicatrização de feridas é um mecanismo que envolve uma sequência de eventos moleculares divididos em três fases com o objetivo de reparo tecidual: fase inflamatória, proliferativa e de remodelagem. A fase de remodelagem tem por objetivo a recuperação da estrutura tecidual marcada por maturação dos elementos e alterações na matriz extracelular, ocorrendo o depósito de proteoglicanas e colágeno (Mendonça \& Coutinho-Netto, 2009).

Neste sentido, destaca-se o uso fototerapia LED (Light Emiting Diodes) com comprimento de onda que varia e 405nm (azul) a 940nm (infravermelho) em uma variedade de situações clínicas pela ativação precoce da fase inflamatória, aumentando significativamente a proliferação dos fibroblastos e redução do infiltrado inflamatório (Nogueira et al., 2014; Meyer et al., 2010).

A palavra laser corresponde ao acrônimo de "light amplification by stimulated emission of radiation" que significa: amplificação de luz por emissão estimulada de radiação. Por definição, o laser é uma radiação eletromagnética que emite luz coerente e colimada e pode ser classificado em lasers de alta potência ou lasers de baixa potência (Filho., 2003; Cavalcanti, Almeida-Barros, Catão, Feitosa \& Lins., 2011). Os lasers de baixa potência são considerados terapêuticos devidos as suas características bioestimulantes para célula, proporcionando aceleração do reparo tecidual devido à ativação mitótica das células epiteliais, produção de colágeno pelos fibroblastos, inibição de mediadores químicos e estímulo a microcirculação local, além de proporcionar um tratamento não-invasivo, não farmacológico e com baixo índice de efeitos colaterais (Lins et al., 2010; Piva, Abreu, Silva \& Nicolau, 2011).

\section{Metodologia}

\section{Delineamento do Estudo}

Tratou-se de uma revisão de literatura do tipo integrativa que é caracterizada pela recuperação de estudos a partir de estratégias de busca especificadas e a síntese de informações e extração de dados de outros estudos que tratem da mesma temática abordada (Souza, Silva \& Carvalho, 2014). A presente revisão baseia-se em uma metodologia qualitativa, sendo importante a interpretação por parte do pesquisador com suas opiniões sobre o fenômeno em estudo (Pereira, Shitsuka, Pareira $\&$ Shitsua., 2018).

\section{Critérios de inclusão e exclusão}

Foram incluídos artigos que abordassem o efeito da fotobiomodulação das luzes vermelha, verde e/ou azul em modelos animais, publicados entre os anos de 2011 a 2020, nos idiomas inglês e português, relacionados à cicatrização de feridas por queimaduras e formação de colágeno no processo de cicatrização.

\section{Formação de informação}

Os artigos foram recuperados a partir das bases de dados: Lilacs (Centro América Latina e Caribe em Ciências da Saúde), Web of Science e PubMed. O levantamento de dados ocorreu no mês de janeiro de 2021.

\section{Estratégia de busca}

A Estratégia de busca utilizada para a pesquisa nas bases de dados se deu pela utilização dos seguintes descritores e palavras-chaves: ("Terapia com luz de baixa intensidade", "Cicatrização", "Queimaduras" e "Fototerapia") em associação de diferentes formas através dos operadores booleanos AND e OR. 
Research, Society and Development, v. 10, n. 3, e3010312855, 2021

(CC BY 4.0) | ISSN 2525-3409 | DOI: http://dx.doi.org/10.33448/rsd-v10i3.12855

\section{Extração de dados}

Foram analisadas as seguintes variáveis: autor, ano, título, objetivo, delineamento do estudo e conclusão.

\section{Resultados}

Foram encontrados 1721 artigos com as palavras chaves utilizadas em associação. Desses, foram excluídos 1703 por não atenderem aos critérios de inclusão. 25 artigos foram excluídos por duplicidade e apenas 18 foram incluídos na revisão integrativa. A Figura 1 descreve os estudos identificados nas bases de dados utilizadas (Pubmed, Web of Science e Lilacs) de acordo com as associações dos descritores utilizados.

Figura 1. Processo de busca, seleção e inclusão de artigos.

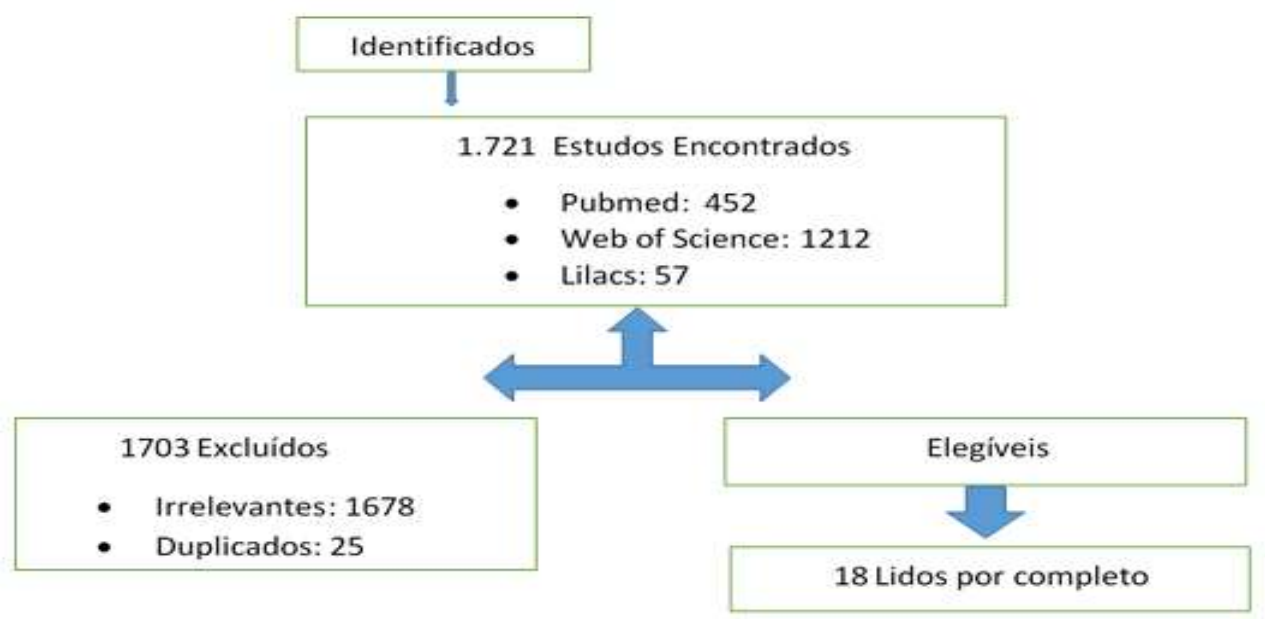

Fonte: Autores

É importante observar a quantidade de artigos elegidos para pesquisa, pois apesar de identificados muitos estudos, poucos estavam relacionados ao tema proposto, indicando que o assunto ainda é pouco abordado em pesquisas acadêmicas. A Figura 2 identifica a estratégia de busca utilizada para pesquisa e os resultados encontrados por base de dados utilizando as palavras chaves em associação. 
Research, Society and Development, v. 10, n. 3, e3010312855, 2021

(CC BY 4.0) | ISSN 2525-3409 | DOI: http://dx.doi.org/10.33448/rsd-v10i3.12855

Figura 2. Estratégia de busca.

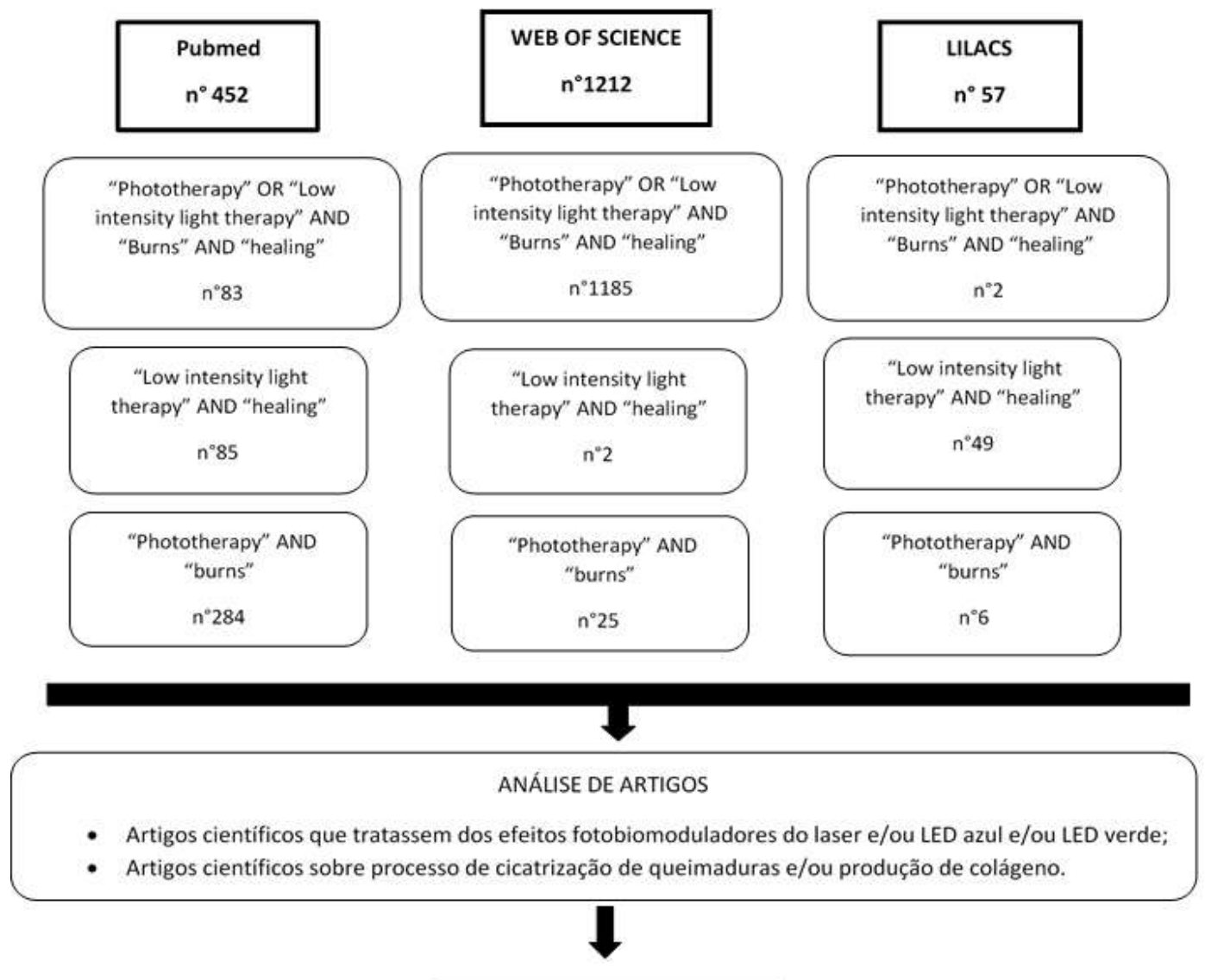

18 artigos selecionados

Fonte: Autores

As associações entre descritores permitiram uma busca abrangente de artigos, da qual 18 foram eleitos conforme análise de tema.

A Tabela 1 identifica os 18 artigos utilizados na revisão integrativa, destacando: Autor/ano, título do artigo, objetivo do estudo, detalhamento do estudo ou protocolo de tratamento utilizado e conclusão. 
Research, Society and Development, v. 10, n. 3, e3010312855, 2021

(CC BY 4.0) | ISSN 2525-3409 | DOI: http://dx.doi.org/10.33448/rsd-v10i3.12855

Tabela 1 Caracterização dos estudos quanto aos objetivos, protocolos de tratamentos e conclusões.

\begin{tabular}{|c|c|c|c|c|}
\hline Autor/Ano & Título & Objetivo & Detalhamento do estudo & Conclusão \\
\hline $\begin{array}{l}\text { Neto JAF } \\
\qquad \text { et al. } \\
2018\end{array}$ & $\begin{array}{l}\text { Effect of blue LED on the } \\
\text { healing process of third- } \\
\text { degree skin burns: clinical } \\
\text { and histological evaluation. }\end{array}$ & $\begin{array}{l}\text { Avaliar os efeitos do diodo } \\
\text { emissor de luz azul (LED) no } \\
\text { processo de cicatrização da } \\
\text { pele por queimaduras de } \\
\text { terceiro grau em ratos por } \\
\text { meio de parâmetros clínicos } \\
\text { e histológicos. }\end{array}$ & $\begin{array}{l}\text { Quarenta ratos Wistar machos } \\
\text { divididos em grupo controle } \\
\qquad(\mathrm{CTR})(\mathrm{n}=20) \mathrm{e} \\
\text { LED azul (AZUL) }(\mathrm{n}=20) \text {. } \\
\text { Aplicação do LED }(470 \mathrm{~nm}, 1 \\
\left.\text { W, } 12,5 \mathrm{~J} / \mathrm{cm}^{2} \text { por ponto, } 28 \mathrm{~s}\right) \\
\text { foi aplicado em quatro pontos da } \\
\left.\text { ferida (total, } 50 \mathrm{~J} / \mathrm{cm}^{2}\right) \text {. }\end{array}$ & $\begin{array}{l}\text { O LED azul, na dosimetria utilizada, } \\
\text { contribui positivamente em etapas } \\
\text { importantes e iniciais do processo de } \\
\text { cicatrização de queimaduras cutâneas } \\
\text { de terceiro grau. }\end{array}$ \\
\hline $\begin{array}{l}\text { Fiorio FB } \\
\qquad \begin{array}{l}\text { et al. } \\
2011\end{array}\end{array}$ & $\begin{array}{l}\text { Effect of incoherent LED } \\
\text { radiation on third-degree } \\
\text { burning wounds in rats }\end{array}$ & $\begin{array}{l}\text { Avaliar os efeitos do LED } \\
\text { vermelho no processo de } \\
\text { reparo da pele de ratos } \\
\text { submetidos à queimadura de } \\
\text { terceiro grau. }\end{array}$ & $\begin{array}{l}\text { Os animais foram divididos em } \\
4 \text { grupos (n 6) da seguinte } \\
\text { forma: grupos L1 e L2 como } \\
\text { ratos queimados tratados com } \\
\text { LED e receberam terapia com } \\
\text { LED ao longo de } 7 \text { e } 15 \text { dias } \\
\text { com intervalos de } 48 \text { horas, } \\
\text { respectivamente; Grupos C1 e } \\
\text { C2 como controle, ratos } \\
\text { queimados não tratados. Foi } \\
\text { usado um LED vermelho ( } 640 \\
\text { nm, } 30 \text { mW) operando com uma } \\
\text { fluência de } 4 \mathrm{~J} / \mathrm{cm}^{2} \text {. A área da } \\
\text { ferida foi medida diariamente } \\
\text { após a irradiação. }\end{array}$ & $\begin{array}{l}\text { O tratamento com LED foi claramente } \\
\text { eficaz na redução do número de células } \\
\text { inflamatórias e na melhoria do processo } \\
\text { de cicatrização em um modelo } \\
\text { experimental de queimaduras de } \\
\text { terceiro grau. }\end{array}$ \\
\hline $\begin{array}{l}\text { Chiarotto GB } \\
\qquad \begin{array}{l}\text { et al. } \\
2014\end{array}\end{array}$ & $\begin{array}{c}\text { Effects of laser irradiation } \\
(670-\mathrm{nm} \text { InGaP and } 830 \text {-nm } \\
\text { GaAlAs) on burn of second- } \\
\text { degree in rats }\end{array}$ & $\begin{array}{l}\text { Este estudo investigou os } \\
\text { efeitos da terapia a laser de } \\
\text { fosfeto de gálio e índio } 670 \\
\mathrm{~nm}(\mathrm{InGaP}) \text { e arsenieto de } \\
\text { alumínio e gálio } 830 \mathrm{~nm} \\
\text { (GaAlAs) em queimaduras } \\
\text { de segundo grau induzida no } \\
\text { dorso de ratos Wistar. }\end{array}$ & $\begin{array}{l}63 \text { ratos machos Wistar foram } \\
\text { anestesiados e queimaduras de } \\
\text { segundo grau foram feitas nas } \\
\text { costas. Os animais foram então } \\
\text { divididos aleatoriamente em três } \\
\text { grupos: controle (C), animais } \\
\text { tratados com InGaP } 670 \mathrm{~nm} \\
\text { laser (LIn), e animais tratados } \\
\text { com laser GaAlAs de } 830 \mathrm{~nm} \\
\text { (LGa). As áreas da ferida foram } \\
\text { removidas após } 2,6,10,14 \text { e } 18 \\
\text { dias de tratamento e submetidos } \\
\text { à análise estrutural e } \\
\text { morfométrica. Os seguintes } \\
\text { parâmetros foram } \\
\text { estudados:número total de } \\
\text { granulócitos e fibroblastos, } \\
\text { número de novos vasos } \\
\text { sanguíneos formados e } \\
\text { porcentagem de colágeno } \\
\text { birrefringente e fibras na área de } \\
\text { reparo. }\end{array}$ & $\begin{array}{l}\text { A terapia a laser InGaP de } 670 \mathrm{~nm} \text { foi } \\
\text { mais eficaz no aumento do número de } \\
\text { fibroblastos. Os diferentes tratamentos } \\
\text { modificaram a expressão de VEGF e } \\
\text { TGF- } \beta 1 \text {, quando comparado com lesões } \\
\text { não irradiadas. Os diferentes tipos de } \\
\text { fontes de luz mostraram efeitos } \\
\text { semelhantes, melhoraram a cicatrização } \\
\text { de queimaduras de segundo grau e } \\
\text { podem ajudar a tratar este tipo de lesão. }\end{array}$ \\
\hline $\begin{array}{l}\text { Fiorio FB } \\
\qquad \begin{array}{l}\text { et al. } \\
2014\end{array}\end{array}$ & $\begin{array}{l}\text { Effect of low-level laser } \\
\text { therapy on types I and III } \\
\text { collagen and inflammatory } \\
\text { cells in rats with induced } \\
\text { third-degree burns. }\end{array}$ & $\begin{array}{c}\text { Investigar os efeitos dos } \\
\text { lasers no processo de reparo } \\
\text { tecidual de queimaduras de } \\
\text { terceiro grau. }\end{array}$ & $\begin{array}{l}\text { Os animais foram divididos em } \\
\text { quatro grupos ( } \mathrm{n}=12 \text { ): controle, } \\
\text { lesão, LLLT } 3 \mathrm{~J} / \mathrm{cm}^{2} \text { e LLLT } 4 \\
\mathrm{~J} / \mathrm{cm}^{2} \text {. Cada grupo foi ainda } \\
\text { dividido em dois subgrupos; os } \\
\text { ratos em um subgrupo foram } \\
\text { mortos em dia } 8 \text { e os do outro, } \\
\text { no dia } 16 \text { após a lesão. Os } \\
\text { animais em LLLT } 3 \mathrm{~J} / \mathrm{cm}^{2} \mathrm{e} \\
\text { LLLT } 4 \mathrm{~J} / \mathrm{cm}^{2} \text { foram irradiados } \\
1 \text { hora após a lesão, e a } \\
\text { irradiação foi repetida a cada } 48 \\
\text { horas. Tratamento a laser ( } 660 \\
\text { nm, } 35 \text { mW) em fluências de } 3 \mathrm{e} \\
4 \mathrm{~J} / \mathrm{cm}^{2} \text { foram usados. Depois } \\
\text { de matar os ratos, fragmentos de } \\
\text { tecido da área queimada foram } \\
\text { removidos para análise }\end{array}$ & $\begin{array}{c}\text { Os grupos tratados com LLLT } \\
\text { mostraram uma diminuição } \\
\text { significativa }(\mathrm{p}<0,05) \text { no número de } \\
\text { células inflamatórias e aumento da } \\
\text { deposição de colágeno em comparação } \\
\text { ao grupo com lesão. Irradiação de laser } \\
\left(\text { ambos } 3 \text { e } 4 \mathrm{~J} / \mathrm{cm}^{2}\right) \text { resultou na } \\
\text { redução do processo inflamatório e } \\
\text { melhorou a deposição de colágeno, } \\
\text { melhorando assim a cicatrização de } \\
\text { queimaduras de terceiro grau. }\end{array}$ \\
\hline
\end{tabular}


Research, Society and Development, v. 10, n. 3, e3010312855, 2021

(CC BY 4.0) | ISSN 2525-3409 | DOI: http://dx.doi.org/10.33448/rsd-v10i3.12855

histológica.

Effect of Low-Power Laser

(LPL) and Light-Emitting Diode (LED) on

Inflammatory Response in

Burn Wound Healing

Silveira PCL

et al.

2016
Catão $M H C V$

et al.

2015

Fekrazad R

Et al.

2017

Catão $\mathrm{MHCV}$

et al.

2016

Effects of red laser, infrared, photodynamic therapy, and green LED on the healing process of third-degree burns: clinical and histological study in rats

Evaluation of Therapeutic Laser Influences on the Healing of Third-degree Burns in Rats According to Different Wavelengths

Green LED light has antiinflammatory effects on burns in rats ED phototherapy in full-
O objetivo deste estudo foi avaliar os efeitos do laser vermelho, infravermelho, terapia fotodinâmica e diodo emissor de luz (LED) verde no processo de cicatrização de queimaduras na pele através da análise clínica e histopatológica em ratos.

O objetivo deste estudo foi investigar o efeito de quatro comprimentos de ond diferentes de laser terapêutico $(405 \mathrm{~nm}, 532 \mathrm{~nm}$, $660 \mathrm{~nm}$ e $810 \mathrm{~nm}$ ) laser na cicatrização de queimaduras de terceiro grau do ponto de vista clínico e patológico em ratos

O objetivo deste estudo foi avaliar os efeitos da luz LED

verde na inflamação em queimaduras de pele: um estudo histológico em ratos.

O objetivo deste estudo foi avaliar os efeitos da fototerapia LED em thickness burns induced by queimaduras de espessura
Investigar as mudanças bioquímicas e moleculares no processo da cicatrização epidérmica de queimaduras após tratamento terapêutico com laser de baixa potência (LPL) e diodo emissor de luz (LED).
Os ratos foram divididos em seis grupos: pele sem lesão (Sham), queimaduras (BWs), BW + LPL de $660 \mathrm{~nm}, \mathrm{BW}+$ LPL de 904 nm, LED BW + 632 nm e LED $\mathrm{BW}+850 \mathrm{~nm}$. O modelo de queimadura foi realizado em placa de cobre a $100^{\circ} \mathrm{C}$, com $10 \mathrm{~s}$ de contato na pele. As irradiações começaram 24 horas após a lesão e foram realizadas diariamente durante 7 dias.

100 animais foram divididos aleatoriamente em cinco grupos:

G1 - controle não tratado

(CTR), G2 - laser vermelho (LVER), G3 - infravermelho (LINF), G4 - terapia fotodinâmica (PDT) e G5 LED verde. A queimadura foi induzida no dorso do rato e o tratamento dos grupos experimentais foi luz vermelha $\left(10 \mathrm{~J} / \mathrm{cm}^{2}, 10 \mathrm{~s}, 40 \mathrm{~mW}\right.$ e $\lambda 660$ $\mathrm{nm})$, infravermelho $\left(10 \mathrm{~J} / \mathrm{cm}^{2}\right.$ $10 \mathrm{~s}, 40 \mathrm{~mW}$ e $\lambda 780 \mathrm{~nm})$ irradiação de LED verde (60 J / $\mathrm{cm}^{2}, 10 \mathrm{~s}, \lambda 520$ e $550 \mathrm{~nm}$ ) e terapia fotodinâmica $(10 \mathrm{~J} /$ $\mathrm{cm}^{2}, 40 \mathrm{~mW}$ e $\left.\lambda 660 \mathrm{~nm}\right), \mathrm{o}$ último combinado com metileno fotossensibilizador azul na concentração de $0,5 \mu \mathrm{g} / \mathrm{mL}$.

60 ratos Wistar machos foram usados. Os animais foram anestesiados e dorsal os cabelos foram raspados e queimaduras de terceiro grau foram criadas com o uso de um carimbo de cobre de $95^{\circ} \mathrm{C}$. As lesões foram irradiadas com densidades de energia de $1,5 \mathrm{~J} / \mathrm{cm}^{2} \mathrm{e}$ densidades de $200 \mathrm{~mW} / \mathrm{cm}^{2}$.

40 ratos foram divididos aleatoriamente em 2 grupos: $\mathrm{G}$ - Controle (CTR) eG2 - Led Verde (LED). Imediatamente após a lesão, luz verde (60 J / $\mathrm{cm}^{2}, 10 \mathrm{~s}, 1520$ a $550 \mathrm{~nm}$ ) foi aplicado em tempo hábil nos quatro pontos coincidentes dos ângulos da ferida e em cada ponto, foi entregue a quantidade de $60 \mathrm{~J} / \mathrm{cm}^{2}$ com tempo de $10 \mathrm{~s}$, totalizando $240 \mathrm{~J} / \mathrm{cm}^{2}$ por sessão com intervalos de 24 horas até o dia anterior ao sacrifício dos animais aos 3,7 , 14 e 21 dias com uma dose leta de anestésico intraperitoneal.

Os animais foram divididos no grupo NT que não recebeu nenhum tratamento e grupo de LED que recebeu irradiação de LED em 685 nm, $220 \mathrm{~mW}$ e 4,5 $\mathrm{J} / \mathrm{cm}^{2}$ durante $40 \mathrm{~s}$ por área total da pele de ratos
Os resultados sugerem que LPL $660 \mathrm{~nm}$ e LED $850 \mathrm{~nm}$ parecem reduzidos nos parâmetros de resposta inflamatória e estresse oxidativo, diminuindo assim a necrose dérmica e aumentando a formação do tecido de granulação, de

fato acelerando a reparação de queimaduras.
Laser vermelho, infravermelho, terapia fotodinâmica e LED verde favoreceram o processo de cicatrização de queimaduras de terceiro grau em ratos.

Lasers terapêuticos com verde, azul, vermelho e comprimentos de onda infravermelhos podem acelerar o processo de cicatrização. Esta tendência é mais óbvia em vermelho e grupos infravermelhos, especialmente após a fase aguda.
A luz LED verde fornece um efeito antiinflamatório em queimaduras na pele de ratos.
A fototerapia LED exerce influência positiva no reparo de queimaduras de espessura total a partir do processo de cicatrização modulado por resposta 
Research, Society and Development, v. 10, n. 3, e3010312855, 2021

(CC BY 4.0) | ISSN 2525-3409 | DOI: http://dx.doi.org/10.33448/rsd-v10i3.12855

et al.

2018

\section{$\mathrm{CO} 2$ laser in rats skin.}

Simões TMS

et al.

2019

Rathnakar B

et al.

2016

Moraes MJ

et al.

2013

Brassolatti P

et al.

2016

Fantinai MS

et al.

2016
Photobiomodulation of red and green lights in the repair process of third-degree skin burns

Photo-biomodulatory response of low-power laser irradiation on burn tissue repair in mice.

Anti-inflammatory effect of low-intensity laser on the healing of third-degree burn wounds in rats
Este estudo teve como objetivo investigar os efeitos do LLLT $(660 \mathrm{~nm})$ em duas fluências diferentes (12,5 J / $\mathrm{cm}^{2}$ e $25 \mathrm{~J} / \mathrm{cm}^{2}$ ) por ponto de aplicação em queimaduras de terceiro grau em ratos.
Comparative Effects of Two

Different Doses of Low-

Level Laser Therapy on

Wound Healing Third-Degree Burns in Rats $\mathrm{nm}$ ) em queimaduras em induzidas por laser de $\mathrm{CO} 2$.
O objetivo deste estudo foi avaliar a fotobiomodulação das luzes vermelha e verde no processo de reparo da pele de queimaduras de terceiro grau em ratos por meio de parâmetros clínicopatológicos e imunohistoquímicos.

\author{
obtidos após 7, 14 e 21 dias de \\ tratamento e submetidos à \\ análise histológica e \\ imunoistoquímica.
}

Sessenta ratos Wistar machos foram divididos em três grupos: controle (CTRL) $(\mathrm{n}=20)$, LED vermelho (VERMELHO) $(\mathrm{n}=$ 20) e LED verde (VERDE) $(\mathrm{n}=$ 20), com subgrupos $(n=5)$ para cada tempo de eutanásia $(7,14$, 21 e 28 dias). Aplicações diárias nos grupos VERMELHO $(\lambda 630$ $\pm 10 \mathrm{~nm}, 300 \mathrm{~mW})$ e VERDE $(\lambda 520 \pm 30 \mathrm{~nm}, 180 \mathrm{~mW})$ foram realizados em quatro pontos da ferida (total $36 \mathrm{~J} / \mathrm{cm}^{2}$ no VERMELHO e $240 \mathrm{~J} / \mathrm{cm}^{2}$ no VERDE).

Animais eram induzidos com uma queimadura de espessura total de $15 \mathrm{~mm}$ e irradiados com várias fluências $(1,2,3,4$ e $6 \mathrm{~J}$ / $\mathrm{cm}^{2}$ ) de cada lcomprimento de onda do laser em estudo com uma taxa de fluência constante $\left(8,49 \mathrm{~mW} / \mathrm{cm}^{2}\right)$. O tamanho da lesão após o tratamento foi monitorado capturando as imagens da ferida em tempo regularintervalos até a cura completa.

Os ratos foram divididos da seguinte forma: grupo 1 controle (tratado com

O presente estudo teve como objetivo comparar o efeito de diferentes densidades energéticas do laser AlGaInP no processo inflamatório e na cicatrização de feridas de queimaduras de terceiro grau em ratos Wistar.

sulfadiazina de prata), grupo 2 recebeu densidade de energia de $3 \mathrm{~J} / \mathrm{cm}^{2}$, e grupo 3 - recebeu $\mathrm{cm}^{2}$.Todos os animais receberam diariamente um curativo oclusivo com prata sulfadiazina

Relatar o efeito
fotobiomodulador dos lasers vermelho $(632,8 \mathrm{~nm}) \mathrm{e}$ densidade de energia de $6 \mathrm{~J} /$ e papaína $8 \%$. A terapia a laser foi realizada alternadamente três vezes por semana. Os animais eram avaliados no $3^{\circ}, 7^{\circ}, 14^{\circ} \mathrm{e}$

$21^{\circ}$ dias após a lesão e sacrificados para o macroscópico, histológico e análise morfométrica

Trinta ratos Wistar divididos em GC, GL12.5 e GL25 foram usados no estudo e submetidos para queimar ferimentos através de um ferro de solda a $150{ }^{\circ} \mathrm{C}$, pressionado nas costas por $10 \mathrm{~s}$. LLLT foi aplicado imediatamente e $2,4,6$ e 8 dias após a indução da ferida.

Os ratos sofreram a indução de queimadura de terceiro grau e foram divididos em quatro grupos da seguinte forma: grupo controle; grupo tratado não diabético; grupo diabético; grupo tratado com diabéticos. Todos os animais receberam
Os resultados sugerem maior potencial da luz verde para estimular a angiogênese nos períodos iniciais e diferenciação miofibroblástica nos períodos finais do reparo de queimaduras cutâneas de terceiro grau. A luz vermelha pode estimular mais reepitelização e retração da ferida, especialmente nas fases de reparo avançado.

Lasers de baixa potência no comprimento de onda $632,8,785$ e 830 $\mathrm{nm}$ mostraram efeitos benéficos na cicatrização de feridas de queimadura, em comparação com controles não tratados.O tratamento promoveu proliferação, neovascularização,

deposição de colágeno, empacotamento mais denso de tecido conjuntivo e epitelização em taxa mais rápida para contrair a ferida.

Uma maior produção de colágeno foi observada em 7 dias e uma maior reepitelização em 21 dias no grupo 3 (6 $\left.\mathrm{J} / \mathrm{cm}^{2}\right)$. Além disso, o último quando em comparação com os outros grupos apresentados macroscopicamente, possui melhor aspecto da cicatriz aos 21 dias com mais granulação tecido e fibrose.

Os resultados sugerem que o uso de 25 $\mathrm{J} / \mathrm{cm}^{2}$ e $1 \mathrm{~J}$ de energia foi mais eficaz na estimulação dos processos celulares envolvidos no reparo de tecidos em queimaduras de terceiro grau em ratos, reduzindo a fase inflamatória e estimulando angiogênese, restaurando assim a microcirculação local que é essencial para a migração celular.

A terapia LBI favoreceu o processo de cicatrização do tecido com dosagem de $3 \mathrm{~J} / \mathrm{cm}^{2}$ na fase inflamatória e com dosagem de $6 \mathrm{~J} / \mathrm{cm}^{2}$ nas fases proliferativa e de remodelação, acelerando a contração da ferida por queimadura e melhorando o processo 
Research, Society and Development, v. 10, n. 3, e3010312855, 2021

(CC BY 4.0) | ISSN 2525-3409 | DOI: http://dx.doi.org/10.33448/rsd-v10i3.12855

Maligieri LAO et

al.

2017

Jin J et al.

2018

Renno ACM

et al.

2011

Oliveira RA et al.
Differing energy densities with laser $670 \mathrm{~nm} \mathrm{InGaP}$ controls inflammation and collagen reorganization in burns

Therapeutic efficacy of early photobiomodulation therapy on the zones of stasis in burns: an experimental rat model study
Este estudo comparou diferentes densidades de energia do laser em queimaduras de segundo grau em ratos com o objetivo de determinar a dosimetria mais eficaz na estimulação do processo de cicatrização. curativos oclusivos durante os dias experimentais. Os animais tratados receberam o seguinte tratamento em dias alternados: diodo laser GaAlAs $(650 \mathrm{~nm} /$ $12 \mathrm{~mW}$ ), fluência de $3 \mathrm{~J} / \mathrm{cm}^{2}$ até o $7^{\circ}$ dia experimental seguido de $6 \mathrm{~J} / \mathrm{cm}^{2}$ do $7^{\circ}$ dia até o dia da eutanásia. As queimaduras eram avaliados morfome-tricamente, macroscopicamente e

microscopicamente aos $3,7,14$,

21 e 30 dias após a indução.

As queimaduras foram induzidas na pele dorsal de 54 animais

divididos em três grupos (n: 18):

1-sem tratamento; 2-lesões irradiadas pelo Fosfeto de ÍndioGálio (InGaP) 670nm (4,93 J / $\mathrm{cm}^{2}$ ) laser; Lesões irradiadas com 3 pelo InGaP-670nm $(9,86$ $\mathrm{J} / \mathrm{cm}^{2}$ ) laser. Amostras foram coletadas aos 2, 10 e 18 dias após a lesão para avaliação.

Trinta ratos foram aleatoriamente divididos em grupos controle (GC) e grupos laser (LG) (15 por grupo). A zona de estase foi formado pela aplicação de um pente de latão quente na pele, resultando em 4

Este estudo teve como objetivo investigar o papel da terapia de fotobiomodulação na prevenção de zonas de estase em queimaduras.
Effect of low-level laser therapy $(660 \mathrm{~nm})$ on the healing of second-degree skin

$$
\text { burns in rats }
$$

O objetivo deste estudo foi investigar os efeitos do laser de $660 \mathrm{~nm}$ na cicatrização de feridas de queimaduras feitas nas costas de ratos.
Low-intensity LED therapy $(658 \mathrm{~nm})$ on burn healing: a series of cases queimaduras retangulares separados por 3 espaços intermediários não queimados. O lado esquerdo foi ferido a laser (LW), enquanto o lado direito continha a ferida protegida (SW). O LW na LG foi imediatamente submetido a terapia de fotobiomodulação, seguido de uma vez ao dia 30 min local sessões de terapia de fotobiomodulação.

Ultrassonografia de pele e angiografia Doppler análises foram usadas para avaliar os estados das zonas de estase em 1, 24 e 96 horas após a lesão para calcular as proporções de necrose nessas áreas.

Foram utilizados 32 ratos Wistar machos. Os animais foram distribuídos aleatoriamente em 2 grupos de 16 animais cada: grupo controle (ratos queimados sem tratamento) e grupo tratado com laser (ratos queimados

tratados com laserterapia). Cada grupo foi dividido em dois subgrupos diferentes, sacrificados em períodos diferentes (subgrupo A: 7 dias após a cirurgia e subgrupo B: 14 dias pós-cirurgia).

Cinco pacientes com queimaduras de pele foram submetidos a fotobiomodulação por LED, diodo GaAsIP, $(\lambda 658$ $\mathrm{nm}$ ) com $40 \mathrm{~mW}, 7 \mathrm{~J} / \mathrm{cm}^{2} \mathrm{em}$ dias alternados. As biópsias de pele queimada foram realizadas de cicatrização.

A densidade de energia de $9,86 \mathrm{~J} / \mathrm{cm}^{2}$ foi mais eficaz na promoção celular respostas relacionadas à

neoangiogênese, diminuindo a inflamação e reorganizando as fibras de colágeno.
A terapia de fotobiomodulação local pode efetivamente melhorar a progressão da lesão na zona de estase.

No entanto, esses efeitos benéficos são limitados aos sítios irradiados diretamente sem efeitos sistêmicos.
A terapia a laser é capaz de promover o reparo da pele de ratos queimados, como resultado da diminuição da área necrótica e uma regulação positiva da imunoexpressão de COX-2 e VEGF.
O objetivo deste estudo foi avaliar os efeitos do LED na cicatrização de queimaduras. 


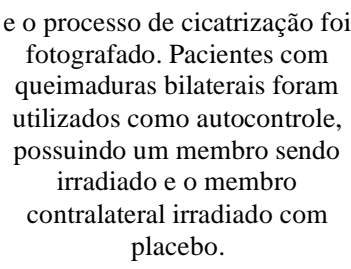

Fonte: Autores.

Na Tabela, é válido observar os protocolos de tratamento seguidos e os resultados consequentes às pesquisas.

\section{Discussão}

Alguns autores avaliaram a produção de colágeno durante o processo de cicatrização das feridas ocasionadas por queimaduras de segundo e terceiro grau quando submetidas a fotobiomodulção.

Fiório, Albertini, Leal-Junior, e Carvalho (2014), em seus estudos sobre os efeitos do laser no processo de reparo em queimaduras de terceiro grau, utilizando laser de comprimento de onda $660 \mathrm{~nm}$ e fluências de 3 e $4 \mathrm{~J} / \mathrm{cm}^{2}$, identificou aumento de colágeno tipo I nos grupos tratados com ambas as doses após 8 dias da lesão, seguido por uma diminuição geral 16 dias após. No entanto, com respeito a deposição do colágeno tipo III no $8^{\circ}$ dia, pode-se observar que o grupo tratado com $3 \mathrm{~J} / \mathrm{cm}^{2}$ obteve valores mais baixos quando comparados com o grupo controle, mas a partir do $16^{\circ}$ o colágeno tipo III foi predominante no grupo de tratamento.

Estes resultados estão de acordo com Maligieri et al. (2017) em seu estudo comparando diferentes densidades de energia do laser InGAP $\lambda=670 \mathrm{~nm}\left(4,93 \mathrm{~J} / \mathrm{cm}^{2} ; 9,86 \mathrm{~J} / \mathrm{cm}^{2}\right)$ para analisar as respostas celulares relacionadas a diminuição da inflamação, angiogênese e reorganização das fibras de colágeno. Em relação as últimas, os dados mostraram diminuição do colágeno tipo III e aumento do tipo I, indicando que a avaliação desses dois tipos tem sido importante na progressão da reparação e que nas fases iniciais predomina colágeno tipo III sendo gradualmente substituído pelo tipo I.

Estudos anteriores mostraram que os lasers regulam a liberação de citocinas responsáveis pela proliferação de fibroblastos e colágeno, melhorando o reparo tecidual (Pereira, Eduardo, Matson \& Marques., 2002) e melhor organização das fibras como observado por Brossolatti et al. (2016), ao estudar os efeitos do laser de comprimento de onda 660nm com fluências de 12,5J (GL12,5) e 25J (GL25). Os resultados destacaram uma evolução mais rápida para a fase proliferativa do grupo tratado com fluência de 25J, além de apresentar melhor organização das fibras de colágeno.

A cicatrização de feridas é um evento complexo e multifatorial que envolve interação do processo de inflamação, formação de tecido de granulação, reepitelização e angiogênese, e deposição de colágeno (Beheregaray et al., 2017; Campos, Borges-Branco, \& Groth., 2007).

Essas informações estão de acordo com o estudo de Fantinati et al. (2016), realizado com ratos diabéticos tratados com laser GaAIAS $\lambda=650 \mathrm{~nm} ; 3 \mathrm{~J} / \mathrm{cm}^{2}$ por 7 dias e $6 \mathrm{~J} / \mathrm{cm}^{2}$ até $30^{\circ}$ dia. Os animais tratados apresentaram significativa contração da ferida, aumento na angiogênese e na quantificação de fibroblastos. A quantidade de colágeno também aumentou em ratos saudáveis e diabéticos com a dose de $6 \mathrm{~J} / \mathrm{cm}^{2}$, favorecendo a fase proliferativa e de remodelação no processo de cicatrização. Chiarotto et al. (2014), através de um estudo analisando diferentes comprimentos de ondas concluiu que o laser InGaP $\lambda=670 \mathrm{~nm}$ aumentou significativamente a quantidade de fibroblastos durante o reparo quando comparado ao laser GaAIAS $\lambda=830 \mathrm{~nm}$.

Esses fibroblastos sofrem influência do fator de crescimento PDGF responsável pela proliferação e ativação destas células. Em seguida é liberado o TGF- $\beta$, que estimula os fibroblastos a produzirem colágeno tipo I e se transformarem em miofibroblastos, que promovem a contração da ferida (Campos et al., 2007). 
Os resultados vão de encontro com Moraes et al. (2013), ao comparar diferentes densidades $\left(3 \mathrm{~J} / \mathrm{cm}^{2} ; 6 \mathrm{~J} / \mathrm{cm}^{2}\right)$ do laser AIGAInP no processo de reparo tecidual pós queimaduras, identificando que a dose de $6 \mathrm{~J} / \mathrm{cm}^{2}$ foi responsável pela maior produção de colágeno, maior epitelização e menor tamanho da ferida aberta quando comparada com o grupo de $3 \mathrm{~J} / \mathrm{cm}^{2}$ sendo esta fluência mais eficaz nas fases iniciais da cicatrização.

Em um outro estudo comparando efeitos de diferentes fluências $\left(1,2,3,4\right.$ e $\left.6 \mathrm{~J} / \mathrm{cm}^{2}\right)$ em queimaduras de camundongos, Rathakar et al. (2016), buscou relatar efeitos do laser vermelho $\lambda=632,8 \mathrm{~nm}$, infravermelho $\lambda=785 \mathrm{~mm}$ e $\lambda=830 \mathrm{~nm}$ no processo de reparo. As fluências foram comparadas para avaliar a retração da ferida, no entanto, todas apresentaram semelhanças nos resultados. $O$ grupo tratado confluência $3 \mathrm{~J} / \mathrm{cm}^{2} ; \lambda=830 \mathrm{~nm}$ obteve destaque em relação a proliferação, neovascularização e deposição de colágeno.

Fekrazad et al. (2017), avaliou o efeito de quatro comprimentos de ondas diferentes: laser verde (G) $\lambda=532 \mathrm{~nm}$; laser azul (B) $\lambda=405 \mathrm{~nm}$; laser vermelho (R) $\lambda=660 \mathrm{~nm}$; laser infravermelho (IR) $\lambda=810 \mathrm{~nm}$; grupo controle (e)= não tratado. Foi observado maior redução da ferida no processo de cicatrização através do laser azul no $7^{\circ}$ dia de tratamento, e o laser vermelho no período restante de estudo. Em contrapartida, após a fase aguda a influência do laser azul diminuiu gradualmente, já a irradiação do laser verde revelou influência positiva no processo de cicatrização após a fase aguda de forma constante, porém, sua efetividade foi menor do que os comprimentos de onda vermelho e infravermelho.

Renno et al. (2011), objetivando investigar os efeitos do laser de $660 \mathrm{~nm}$ na cicatrização de feridas de queimaduras feitas nas costas de ratos, utilizou 32 ratos Wistar machos. Os animais foram distribuídos aleatoriamente em 2 grupos: grupo controle sem tratamento e grupo tratado com laserterapia. Os resultados foram de encontro aos estudos relacionados, avaliando que a terapia a laser é capaz de promover o reparo da pele de ratos queimados, como resultado da diminuição da área necrótica. Jin J et al. (2018), também encontrou semelhanças em seus estudos ao avaliar que a melhora do fluxo sanguíneo ocasionado pela fotobiomodulação está entre os principais mecanismos pelos quais essa terapia melhora o progresso da necrose.

Além da fototerapia laser, o LED também é uma alternativa em potencial no reparo de queimaduras cutâneas, no entanto, ainda são poucos os estudos dos efeitos do LED em processos de cicatrização. A fotoestimulação decorrente dessa luz atua sobre a célula na permeabilidade, sobre as mitocôndrias estimuladoras, na síntese de ATP e nas proteínas como colágeno e a elastina. Esta luz age também como antimicrobiano e antiinflamatório, dependendo do comprimento de onda e, por isso, os LEDs são indicados para as mais variadas afecções inflamatórias (Meyer et al., 2010).

Em sua pesquisa a respeito dos benefícios da fotobiomodulação na cicatrização de queimaduras cutâneas, Fiorio et al. (2011), avaliou o LED vermelho $\lambda=640 \mathrm{~nm} ; 4 \mathrm{~J} / \mathrm{cm}^{2}$. Os resultados foram a diminuição de células inflamatórias no grupo tratado com LED e a redução do tamanho da lesão.

Indo de encontro aos resultados propostos por demais autores já relatados, Silveira et al. (2016), analisando as mudanças celulares no processo de cicatrização por aplicação de diferentes comprimentos de onda da luz laser e LED, sugeriu que a irradiação com laser $\lambda=660 \mathrm{~nm}$ e LED $\lambda=850 \mathrm{~nm}$ também parece ter efeitos positivos na redução do tamanho da lesão, além de apresentarem melhor resposta a inflamação e ao desenvolvimento de papilas dérmicas. Ambos comprimentos de onda da luz Led demonstraram melhor organização do tecido e formação de tecido de granulação. Esse tecido expressa 30 a $40 \%$ de colágeno do tipo III, sendo considerado colágeno imaturo (Robson, Steed \& Franz., 2001), o que, de fato, acelera a cicatrização. Em contrapartida, o laser $\lambda=904 \mathrm{~nm}$ parece não ter efeitos significativos sob a lesão.

Ademais, a análise de Oliveira, Boson, Portela, Filho, e de Oliveira (2017), sob pacientes submetidos a LED diodo $\lambda=$ $658 \mathrm{~nm}$ apresentou resultados satisfatórios de analgesia, redução de prurido e diminuição da quantidade de exsudato, contribuindo na diminuição das características inflamatórias da lesão. 
Em relação a formação do colágeno, Catão, Nonaka, de Albuquerque Júnior, Bento, e Costa (2015), buscaram avaliar os efeitos do laser (LVER) vermelho infravermelho (LINF), terapia (PDT) e luz LED verde no processo de cicatrização de queimaduras (Luz vermelha $\lambda=660 \mathrm{~nm}, 10 \mathrm{~J} / \mathrm{cm}^{2}$; Infravermelho $\lambda=780 \mathrm{~nm}, 10 \mathrm{~J} / \mathrm{cm}^{2}$; LED verde $\lambda=520 \mathrm{~nm}$ e $\lambda=550 \mathrm{~nm}, 60 \mathrm{~J} /$ $\mathrm{cm}^{2}$; terapia fotodinâmica $\lambda=600 \mathrm{~nm}, 10 \mathrm{~J} / \mathrm{cm}^{2}$ ). Para o laser infravermelho em 14 dias, houve maior formação de colágeno em comparação com os outros grupos seguido pelo laser vermelho. Em 14 dias o colágeno tipo I predominou em $60 \%$ nos grupos tratados, enquanto no grupo controle essa porcentagem caiu para 20\% dos animais. Em 21 dias todos animais do grupo tratado apresentaram predominância do colágeno tipo I enquanto o grupo controle possuía $60 \%$ maior retração da ferida em tratamentos com LVER e LINF.

Esse colágeno tipo I é o mais frequente, sintetizado pelos fibroblastos, e é mais predominante em ossos e tendões. O tipo III é mais comumente encontrado em tecidos moles, como vasos sanguíneos, derme e fáscia. A derme saudável contém aproximadamente $80 \%$ de colágeno tipo I e $20 \%$ de colágeno tipo III (Robson et al., 2001).

Simões, Fernandes Neto, de Oliveira, Nonaka e Catão (2019), objetivaram avaliar a fotobiomodulação das luzes vermelhas e verdes no processo de reparo da pele após queimadura de terceiro grau utilizando o LED vermelho $\lambda=630 \mathrm{~nm}$ e o LED verde $\lambda=520 \mathrm{~mm}$. Aos 14 e 21 dias houve maior retração das áreas das feridas do grupo vermelho quando comparado com o verde. Após 21 dias $80 \%$ dos animais do grupo verde não atingiram a reepitelização, aos 28 dias esta foi mais avançada no grupo vermelho. $O$ índice de angiogênese da luz verde foi superior nas fases iniciais de 7 a 14 dias em comparação com vermelho e com o grupo controle, sugerindo maior potencial para estimular a proliferação de vasos.

Resultados semelhantes foram obtidos por Melo et al. (2018), com LED $\lambda=685 \mathrm{~nm}$. Nesse estudo observou-se aumento do número de vasos sanguíneos, proliferação epitelial moderada e fibroblastos, indicando que o LED potencializa a síntese da matriz de tecido conjuntivo.

Esses resultados estão de acordo com os efeitos da luz LED azul no processo de cicatrização da pele por queimadura de terceiro grau em ratos com LED ( $\lambda=470 \mathrm{~nm} ; 12,55 / \mathrm{cm}^{2} ; 28$ segundos /pontos) avaliados por Fernandes Neto, Nonaka e Catão (2018). O estudo observou que a fototerapia foi eficaz em etapas iniciais e importantes do reparo tecidual, a exemplo da reepitelização e angiogênese. A angiogênese é estimulada pelo fator de necrose tumoral alfa (TNF- $\alpha$ ), e é caracterizada pela migração de células endoteliais e formação de capilares, essencial para a cicatrização adequada (Campos et al., 2007). Em relação a neovascularização, houve um aumento estatisticamente significativo em 7 dias no grupo de tratamento (Neto et al., 2018).

De acordo com um estudo realizado por Catão, Costa, Nonaka, Albuquerque Junior, e Costa (2016), a luz LED verde $\lambda=550 \mathrm{~nm} ; 60 \mathrm{~J} / \mathrm{cm}^{2}$, diminui quantitativamente as células inflamatórias a partir do $7^{\circ}$ dia, favorecendo a cicatrização de queimaduras na pele de ratos.

\section{Conclusão}

Após a análise dos artigos selecionados verificou-se que a fotobiomodulação das luzes vermelha, azul e verde no processo de reparo de queimaduras cutâneas mostrou resultados satisfatórios principalmente em relação a produção de colágeno, angiogênese, redução da inflamação, redução do tamanho da lesão e estímulo de fibroblastos. Porém, os estudos em relação as luzes azul e verde no processo de reparo de queimaduras ainda são escassos.

Diante disso, é importante que mais estudos pré-clínicos e clínicos bem construídos sejam realizados, a fim de ampliar cada vez mais o conhecimento sobre essas luzes em relação a produção de colágeno e ao processo de reparo tecidual. 
Research, Society and Development, v. 10, n. 3, e3010312855, 2021

(CC BY 4.0) | ISSN 2525-3409 | DOI: http://dx.doi.org/10.33448/rsd-v10i3.12855

\section{Referências}

Beheregaray, W. K., Gianotti, G. C., Oliveira, F., Terraciano, P., Bianchi, S., Vidor, S., Marcolan, C. F., Contesini, E. A., \& Cirne-Lima, E. O. (2017). Células-tronco mesenquimais aplicadas nas fases inflamatória e proliferativa da cicatrização de feridas cutâneas. Arquivo Brasileiro de Medicina Veterinária e Zootecnia, 69(6), 1591-1600. https://dx.doi.org/10.1590/1678-4162-9461.

Brassolatti, P., Bossini, P. S., Oliveira, M. C., Kido, H. W., Tim, C. R., Almeida-Lopes, L., De Avó, L. R., Araújo-Moreira, F. M., \& Parizotto, N. A. (2016). Comparative effects of two different doses of low-level laser therapy on wound healing third-degree burns in rats. Microsc Res Tech, 79(4), 313-320. https://doi.org/10.1002/jemt.22632.

Campos, A. C. L., Borges-Branco, A., \& Groth, A. K. (2007). Cicatrização de feridas. ABCD. Arquivos Brasileiros de Cirurgia Digestiva, 20(1), 51-58. http://dx.doi.org/10.1590/S0102-67202007000100010.

Castilho Filho, T. (2003). Avaliação da ação da radiação laser em baixa intensidade no processo de ósseo-integração de implantes de titânio inseridos em tíbia de coelhos. Dissertação de Mestrado, Instituto de pesquisa energéticas nucleares da Faculdade de Odontologia da Universidade de São Paulo; São Paulo, SP, Brasil.

Catão M. H. C. V., Nonaka, C. F. W., de Albuquerque Júnior, R. L. C., Bento, P. M., \& Costa, R. O. (2015). Effects of red laser, infrared, photodynamic therapy, and green LED on the healing process of third-degree burns: clinical and histological study in rats. Lasers in Medical Science, 30(1), 421-428. https://doi.org/10.1007/s10103-014-1687-0.

Catão, M. H. C. V., Costa R. O., Nonaka C. F. W., Albuquerque Junior, R. C., \& Costa, I. R. R. S. (2016). Green LED light has anti-inflammatory effects on burns in rats. Burns, 42(2), 392-396. https://doi.org/10.1016/j.burns.2015.07.003.

Cavalcanti, T. M., Almeida-Barros, R. Q., Catão, M. H. C. V., Feitosa, A. P. A., \& Lins, R. D. A. U. (2011). Conhecimento das propriedades físicas e da interação do laser com os tecidos biológicos na odontologia. Anais Brasileiros de Dermatologia, 86(5), 955-960. https://doi.org/10.1590/S036505962011000500014

Chiarotto, G. B., Neves, L. M. G., Esquisatto, M. A. M., Amaral M. E. C., Santos G. M. T., \& Mendonça F. A. S. (2014). Effects of laser irradiation (670-nm InGaP and 830-nm GaAlAs) on burn of second-degree in rats. Lasers in Medical Science, 29(5), 1685-1693. https://doi.org/10.1007/s10103-014-1573-9.

de Mendonça, R. J., \& Coutinho-Netto, J. (2009). Aspectos celulares da cicatrização. Anais Brasileiros de Dermatologia, 84(3), 257262. https://doi.org/10.1590/S0365-05962009000300007.

de Moraes, J. M., Mendonça, D. E. O., Moura, V. B. L., Oliveira, M. A. P., Afonso, C. L., Vinaud, M. C., Bachion, M. M., \& Lino Junior, R. Jr. (2013). Antiinflammatory effect of low-intensity laser on the healing of third-degree burn wounds in rats. Lasers in Medical Science, 28(4), 1169-1176. https://doi.org/10.1007/s10103-012-1213-1.

de Oliveira, R. A., Boson, L. L. B., Portela, S. M. M., Maia Filho, A. L. M., \& de Oliveira, D. S. (2017). Low-intensity LED therapy (658 nm) on burn healing: a series of cases. Lasers in Medical Science, 33(4), 729-735. https://doi.org/10.1007/s10103-017-2399-z.

Fantinati, M. S., Mendonça, D. E. O., Fantinati, A. M. M., Barbosa, D. A., Araújo, L. C., Afonso, C. L., Vinaud, M. C., \& Lino Júnior, R. S. (2016). Activity of low level laser therapy on burning wounds in diabetic rats. Rev Bras Queimaduras, 15(1), 42-49.

Fekrazad, R., Nikkerdar, A., Khojasteh J., Kalhori, K. A, M., Abbas, F. M., \& Vahid, F. S. (2017). Evaluation of therapeutic laser influences on the healing of third-degree burns in rats according to different wavelengths. Journal of Cosmetic and Laser Therapy, 19(4), 232-236. https://doi.org/10.1080/14764172.2017.1288255.

Fernandes Neto, J. A., Nonaka C. F. W., \& Catão M. H. C. V. (2018). Effect of blue LED on the healing process of third-degree skin burns: clinical and histological evaluation. Lasers in Medical Science, 34(4), 721-728. https://doi.org/10.1007/s10103-018-2647-x.

Fiório, B. F., Silveira Junior L., Munin E., Lima C. J., Fernandes K. P. S., R., Mesquita-Ferrari R. A., Carvalho, P. T. C., Lopes-Martins R. A. B., Aimbire F., \& Carvalho R. A. (2011). Effect of incoherent LED radiation on third-degree burning wounds in rats. Journal of Cosmetic and Laser Therapy, 13(6), 315-322. https://doi.org/10.3109/14764172.2011.630082.

Fiório, F. B., Albertini, R., Leal-Junior, E. C. P., \& Carvalho P. T. C. (2014). Effect of low-level laser therapy on types I and III collagen and inflammatory cells in rats with induced third-degree burns. Lasers in Medical Science, 29(1), 313-319. https://doi.org/10.1007/s10103-013-1341-2.

Jin, J., Zheng, X., He, F., Zhang, Y., Zhou, H., Luo, P., Hu, X., \& Xia, Z. (2018). Therapeutic efficacy of early photobiomodulation therapy on the zones of stasis in burns: An experimental rat model study. Wound Repair and Regeneration, 26(6), 426-436. https://doi.org/10.1111/wrr.12661.

Lins, R. D. A. U., Dantas, E. M., Lucena, K. C. R., Catão, M. H. C. V, Granville-Garcia, A. F, \& Carvalho Neto, L. G. (2010). Efeitos bioestimulantes do laser de baixa potência no processo de reparo. Anais Brasileiros de Dermatologia, 85(6), 849-855. https://doi.org/10.1590/S0365-05962010000600011.

Maligieri, L. A. O., Neves, L. M. G., de Morais, D. T., Domingues, R. F., de Aro, A. A., Pimentel, E. R., do Amaral, M. E. C., Esquisatto, M. A. M., Dos Santos, G. M. T., \& Mendonça, F. A. S. (2017). Differing energy densities with laser 670nm InGaP controls inflammation and collagen reorganization in burns. Burns, 43(7), 1524-1531. https://doi.org/10.1016/j.burns.2017.04.008.

Melo, M. S., Alves, L. P., Fernandes, A. B. Carvalho, H., C., de Lima, C. J., Munin, E., Gomes, M. F., Salgado, M. A. C., \& Zângaro, R. A. (2018). LED phototherapy in full-thickness burns induced by $\mathrm{CO}_{2}$ laser in rats skin. Lasers in Medical Science, 33, 1537-1547. https://doi.org/10.1007/s10103-018-2515-8.

Meyer, P. F., Araújo, H. G., Carvalho, M. G. F., Tatum, B. I. S., Fernandes, I. C. A. G., Ronzio, O. A., \& Pinto, M. V. M. (2010). Avaliação dos efeitos do LED na cicatrização de feridas cutâneas em ratos Wistar. Fisioterapia Brasil, 11(6), 428-432. http://dx.doi.org/10.33233/fb.v11i6.1592. 
Research, Society and Development, v. 10, n. 3, e3010312855, 2021

(CC BY 4.0) | ISSN 2525-3409 | DOI: http://dx.doi.org/10.33448/rsd-v10i3.12855

Nogueira, V. C., Coelho, N. P. M. F., de Barros, T. L., Silva, S. M. M. S., Martins, M., \& Arisawa, E. A. L. (2014). Biomodulation effects of LED and therapeutic ultrasound combined with semipermeable dressing in the repair process of cutaneous lesions in rats. Acta cir. Bras, 29(9), 588-595. https://doi.org/10.1590/S0102-8650201400150006.

Pereira, A. N., Eduardo, C. P., Matson, E., \& Marques, M. M. (2002). Effect of low-power laser irradiation on cell growth and procollagen synthesis of cultured fibroblastos. Lasers in Surgery and Medicine, 31(4), 263-267. https://doi.org/10.1002/lsm.10107.

Pereira, A. S., Shitsuka, D. M., Parreira, F. J., \& Shitsuka, R. (2018). Metodologia da pesquisa científica: UAB/NTE/UFSM.

Piva, J. A. A. C., Abreu, E. M. C., Silva, V., \& Nicolau, R. A. (2011). Ação da terapia com laser de baixa potência nas fases iniciais do reparo tecidual: princípios básicos. An. Bras. Dermatol, 86(5), 947-954. https://doi.org/10.1590/S0365-05962011000500013.

Rathnakar, B., Rao, B. S. S., Prabhu, V., Chandra, S., Rai, S., Rao, A. C. K., Sharma, M., Gupta, P. K., \& Mahato, K. K. (2016). Photo-biomodulatory response of low-power laser irradiation on burn tissue repair in mice. Lasers in Medical Science, 31(9), 1741-1750. https://doi.org/10.1007/s10103-016-20442 .

Renno, A. C., Iwama, A. M., Shima, P., Fernandes, K. R., Carvalho, J. G., De Oliveira P., \& Ribeiro, D. A. (2011). Effect of low-level laser therapy (660 nm) on the healing of second-degree skin burns in rats. J Cosmet Laser Ther, 13(5), 237-242. https://doi.org/10.3109/14764172.2011.606466.

Robson, M. C., Steed, D. L., \& Franz, M. G. (2001). Wound healing: biologic features and approaches to maximize healing trajectories. Curr Probl Surg, 38(2), 72-140. https://doi.org/10.1067/msg.2001.111167.

Silveira, P. C. L., Ferreira, K. B., da Rocha, F. R., Pieri B. L. S., Pedroso G. S., de Souza C. T., Nesi, R. T., \& Pinho, R. A. (2016). Effect of Low-Power Laser (LPL) and Light-Emitting Diode (LED) on inflammatory response in burn wound healing. Inflammation, 39(4), 1395-1404. https://doi.org/10.1007/s10753016-0371-x.

Simões, T. M. S., Fernandes Neto, J. A., de Oliveira, T. K. B., Nonaka C. F. W., \& Catão, M. H. C. V. (2019). Photobiomodulation of red and green lights in the repair process of third-degree skin burns. Lasers in Medical Science, 35(1), 51-61. https://doi.org/10.1007/s10103-019-02776-7. 